\title{
Optimal Polynomial Decay to Coupled Wave Equations and Its Numerical Properties
}

\author{
R. F. C. Lobato, S. M. S. Cordeiro, M. L. Santos, and D. S. Almeida Júnior \\ Department of Mathematics, Federal University of Para, Augusto Corrêa Street, 01, 66075-110 Belem, PA, Brazil \\ Correspondence should be addressed to D. S. Almeida Júnior; dilberto@ufpa.br
}

Received 28 November 2013; Revised 1 April 2014; Accepted 19 May 2014; Published 22 July 2014

Academic Editor: Roberto Barrio

Copyright (C) 2014 R. F. C. Lobato et al. This is an open access article distributed under the Creative Commons Attribution License, which permits unrestricted use, distribution, and reproduction in any medium, provided the original work is properly cited.

In this work we consider a coupled system of two weakly dissipative wave equations. We show that the solution of this system decays polynomially and the decay rate is optimal. Computational experiments are conducted in the one-dimensional case in order to show that the energies properties are preserved. In particular, we use finite differences and also spectral methods.

\section{Introduction}

This paper is concerned with the polynomial stability and the optimal rate of decay of the $C_{0}$-semigroup of the coupled system of two wave equations:

$$
\begin{gathered}
\left.u_{t t}-\Delta u+u_{t}+\alpha v=0 \quad \text { in } \Omega \times\right] 0, \infty[, \\
\left.v_{t t}-\Delta v+\alpha u=0 \quad \text { in } \Omega \times\right] 0, \infty[, \\
u=v=0 \quad \text { on } \Gamma \times] 0, \infty[, \\
(u(x, 0), v(x, 0))=\left(u_{0}, v_{0}\right), \\
\left(u_{t}(x, 0), v_{t}(x, 0)\right)=\left(u_{1}(x), v_{1}(x)\right), \\
\text { in } \Omega,
\end{gathered}
$$

where $\Omega$ is an open bounded set of $\mathbb{R}^{n}$ with smooth boundary $\Gamma$. Here $\alpha$ is a small real positive constant.

This problem is motivated by an analogous problem in ordinary differential equations for coupled oscillators and has potential application in isolation of objects from outside disturbances. As an example in engineering, rubber-like materials are used to absorb vibration or shield structures from vibration. Modeling of structures such as beam or plates sandwiched with rubber or similar materials will lead to equations similar to those (1)-(2).

The exponential stability of coupled system of wave equations has been studied by Najafi et al. [1] in the case of two linear boundary feedback and by Komornik Rao [2] in the case of two nonlinear boundary feedback.

We should mention that this initial boundary value problem (1)-(4) has been studied by many authors. For example, in [3] Alabau Boussoira proved that the energy of associated coupled weakly dissipative system decays polynomially as $t^{-1}$ and this decay rate can improve since the initial data are more regular. In [4] Alabau Boussoira et al. showed the decay rates for an abstract coupled weakly dissipative system of using the same ideas introduced in [3]. For the case of the system (1)(4) we can also cite the work of Santos et al. [5] where the authors proved $t^{-1}$ polynomial decay and this decay rate can improve since the initial data are more regular. The question we are interested in is to study optimal polynomial decay rates to the system (1)-(4). That is, we prove that the associated semigroup decays at a rate $t^{-1 / 2}$ and it is optimal. This work improves the results in [3-5]. Our result on the polynomial stability is based on Theorem 2.4 in [6].

Naturally, the system (1)-(4) is dissipative. Indeed, the energy of the solutions defined by

$$
\begin{aligned}
E(t):= & \frac{1}{2} \int_{\Omega} u_{t}^{2} d x+\frac{1}{2} \int_{\Omega}|\nabla u|^{2} d x+\frac{1}{2} \int_{\Omega} v_{t}^{2} d x \\
& +\frac{1}{2} \int_{\Omega}|\nabla v|^{2} d x+\alpha \int_{\Omega} u v d x
\end{aligned}
$$

is decreasing in $t \in(0, \infty)$, since

$$
\frac{d}{d t} E(t)=-\int_{\Omega} u_{t}^{2} d x \leq 0 .
$$


To verify the asymptotic behavior of systems like (1)-(4), only the work of Najafi [7] treated numerically the problem of lack of exponential decay to a system of two coupled wave equations. Here, we use two numerical approaches: one is in finite differences and in the other one we use the spectral methods. In particular, we explore the properties of numerical energy associated with a particular discretization in finite differences.

We conclude this introduction with an outline of the paper. In Section 2, we show the existence of solutions for the system (1)-(4). In the Section 3 we showed that the system (1)-(4) is polynomially stable with optimal decay rate. Section 4 is dedicated with the numerical aspects. We derive a numerical energy and we show that it preserves the conservation and dissipation laws. We use also spectral methods to see the behavior of the numerical eigenvalues when the damping is weak. Finally, in Section 5, we finished this work with a conclusion.

\section{The Semigroup Setting}

In this section we will study the existence and uniqueness of strong and global solutions for the system (1)-(4) using the semigroup techniques.

Let us denote by $\mathscr{H}=H_{0}^{1}(\Omega) \times L^{2}(\Omega) \times H_{0}^{1}(\Omega) \times L^{2}(\Omega)$ the Hilbert space with internal product given by

$$
\begin{array}{r}
\langle U, V\rangle_{\mathscr{H}}=\int_{\Omega}\left[\nabla u_{1} \cdot \nabla v_{1}+u_{2} v_{2}+\nabla u_{3} \cdot \nabla v_{3}\right. \\
\left.+u_{4} v_{4}+\alpha\left(u_{1} v_{3}+u_{3} v_{1}\right)\right] d x
\end{array}
$$

where $U=\left(u_{1}, u_{2}, u_{3}, u_{4}\right)^{T}$ and $V=\left(v_{1}, v_{2}, v_{3}, v_{4}\right)^{T}$.

Let us consider the unbounded operator $\mathscr{A}$ in the energy space $\mathscr{H}$ with

$$
\begin{aligned}
D(\mathscr{A})= & H^{2}(\Omega) \cap H_{0}^{1}(\Omega) \times H_{0}^{1}(\Omega) \\
& \times H^{2}(\Omega) \cap H_{0}^{1}(\Omega) \times H_{0}^{1}(\Omega),
\end{aligned}
$$

defined by

$$
\mathscr{A}=\left[\begin{array}{cccc}
0 & I & 0 & 0 \\
\Delta & -I & -\alpha I & 0 \\
0 & 0 & 0 & I \\
-\alpha I & 0 & \Delta & 0
\end{array}\right]
$$

Putting $\varphi=u_{t}$ and $\psi=v_{t}$, (1) and (2) can be written as the following initial value problem:

$$
\begin{aligned}
& \frac{d U}{d t}=\mathscr{A} U, \\
& U(0)=U_{0},
\end{aligned}
$$

with $U=(u, \varphi, v, \psi)^{T}$ and $U_{0}=\left(u_{0}, u_{1}, v_{0}, v_{1}\right)^{T}$. Using the internal product (7), we obtain

$$
\mathscr{R} e\langle\mathscr{A} U, U\rangle_{\mathscr{H}}=-\int_{\Omega}|\varphi|^{2} d x \leq 0 .
$$

Thus, $\mathscr{A}$ is a dissipative operator and therefore, using the Lumer-Phillips Theorem (see [8], Theorem 4.3), the operator $\mathscr{A}$ generates a $C_{0}$-semigroup of contractions $S(t)=e^{\mathscr{A} t}$ on $\mathscr{H}$.

Thus, we have the following result.

Theorem 1. Let one assume that $U_{0} \in D(\mathscr{A})$; then there exists a unique solution $U=(u, \varphi, v, \psi)$ to system (1)-(4) satisfying

$$
U \in C\left(\mathbb{R}^{+} ; D(\mathscr{A})\right) \cap C^{1}\left(\mathbb{R}^{+} ; \mathscr{H}\right) .
$$

\section{Polynomial Decay and Optimal Result}

To study the asymptotic behavior of the semigroup associated with (1)-(4), let us consider the spectral problem:

$$
\begin{gathered}
-\Delta w_{v}=\lambda_{v} w_{v} \quad \text { in } \Omega, \\
w_{v}=0 \quad \text { on } \Gamma,
\end{gathered}
$$

where

$$
\lim _{\nu \rightarrow \infty} \lambda_{\nu}=+\infty
$$

Theorem 2. Let $S(t)$ be $C_{0}$-semigroup of contractions generated by $\mathscr{A}$. Then it follows that $S(t)$ is not exponentially stable.

Proof. Our main tool is to use Prüss' result [9], which states that a semigroup $e^{\mathscr{A} t}$ is exponentially stable if and only if the following conditions hold:

$$
i \mathbb{R} \subset \rho(\mathscr{A}) \quad \text { (resolvent set), }
$$

$$
\exists K>0, \forall U \in D(\mathscr{A}), \forall \lambda \in \mathbb{R}: \quad\left\|(i \lambda I-\mathscr{A})^{-1}\right\|_{\mathscr{H}} \leq K .
$$

This way, let us consider the resolvent equation

$$
(i \lambda I-\mathscr{A}) U=F, \quad \text { with } \lambda \in \mathbb{R}, F \in \mathscr{H} \text {; }
$$

that is,

$$
\begin{gathered}
i \lambda u-\varphi=f^{1}, \\
i \lambda \varphi-\Delta u+\alpha v+\varphi=f^{2}, \\
i \lambda v-\psi=f^{3}, \\
i \lambda \psi-\Delta v+\alpha u=f^{4} .
\end{gathered}
$$

Let us take $f^{1}=f^{3}=0$ and $f^{2}=f^{4}=w_{\nu}$. We look for solution of the form $u=a w_{v}, v=b w_{v}, \varphi=c w_{\gamma}$, and $\psi=d w_{v}$, with $a, b, c, d \in \mathbb{C}$. From (19)-(21), we get $a$ and $b$ satisfying

$$
-\lambda^{2}(a+b) w_{\nu}+(a+b) \lambda_{\nu} w_{\nu}+\alpha(a+b) w_{v}+c w_{\nu}=2 w_{\nu}
$$

Now, choosing $\lambda=\sqrt{\lambda_{v}+\alpha}$, and using the above equation, we obtain $c=2$, so we have

$$
\begin{gathered}
a=\frac{-2 i}{\sqrt{\lambda_{v}+\alpha}}, \quad b=\frac{2 i}{\sqrt{\lambda_{v}+\alpha}}+\frac{1}{\alpha}, \\
d=\frac{-2 \alpha+i \sqrt{\lambda_{v}+\alpha}}{\alpha} .
\end{gathered}
$$


Therefore, we have

$$
\begin{gathered}
u=\frac{-2 i}{\sqrt{\lambda_{\nu}+\alpha}} w_{\nu}, \quad v=\left(\frac{2 i}{\sqrt{\lambda_{v}+\alpha}}+\frac{1}{\alpha}\right) w_{\nu} \\
\varphi=2 w_{v}, \quad \psi=\frac{-2 \alpha+i \sqrt{\lambda_{v}+\alpha}}{\alpha} w_{v} .
\end{gathered}
$$

Now we claim that

$$
\|U\|_{\mathscr{H}} \longrightarrow+\infty, \quad \text { as } v \longrightarrow+\infty \text {. }
$$

In fact, using (7) and (24) and noting that

$$
\lim _{\nu \rightarrow \infty} \lambda_{\nu}=\infty
$$

we conclude that

$$
\|U\|_{\mathscr{H}}^{2} \geq \int_{\Omega}\left|\left(\frac{-2 \alpha+i \sqrt{\lambda_{\nu}+\alpha}}{\alpha} w_{v}\right)\right|^{2} d x \longrightarrow \infty .
$$

Recalling that

$$
i \lambda U-\mathscr{A} U=F \Longleftrightarrow U=(i \lambda I-\mathscr{A})^{-1} F,
$$

it follows from (27) and (16) that $S(t)$ is not exponentially stable.

In order to show the polynomial decay from semigroup associated with the system (1)-(4), first let us consider the product in $\mathscr{H}$ of $U=(u, \varphi, v, \psi)^{T} \in D(\mathscr{A})$ with the resolvent equation of $\mathscr{A}$; that is,

$$
i \lambda\|U\|_{\mathscr{H}}^{2}-(\mathscr{A} U, U)_{\mathscr{H}}=(F, U)_{\mathscr{H}} .
$$

Now, noting that

$$
\int_{\Omega}|\varphi|^{2} d x=-(\mathscr{A} U, U)^{\mathscr{H}}
$$

we get

$$
i \lambda\|U\|_{\mathscr{H}}^{2}+\int_{\Omega}|\varphi|^{2} d x=(F, U)_{\mathscr{H}}
$$

Then taking the real part, we obtain

$$
\int_{\Omega}|\varphi|^{2} d x \leq\|U\|_{\mathscr{H}}\|F\|_{\mathscr{H}}
$$

Lemma 3. The strong solution of the system (1)-(4) given by Theorem 1 satisfies

$$
\begin{gathered}
\int_{\Omega}|\nabla u|^{2} d x+\int_{\Omega}|\nabla v|^{2} d x+\alpha \int_{\Omega}(u \bar{v}+v \bar{u}) d x \\
\leq \int_{\Omega}|\psi|^{2} d x+K\|U\|_{\mathscr{H}}\|F\|_{\mathscr{H}},
\end{gathered}
$$

where $K$ is a positive constant.
Proof. Multiplying equalities (19) and (21) by $\bar{u}$ and $\bar{v}$, respectively, integrating on $\Omega$, and summing up the result we get

$$
\begin{aligned}
& \underbrace{i \lambda \int_{\Omega} \varphi \bar{u} d x}_{:=I_{1}}+\int_{\Omega}|\nabla u|^{2} d x+\alpha \int_{\Omega} v \bar{u} d x \\
& \quad+\underbrace{i \lambda \int_{\Omega} \psi \bar{v} d x}_{:=I_{2}}+\int_{\Omega}|\nabla v|^{2} d x+\alpha \int_{\Omega} \int u \bar{v} d x \\
& =\int_{\Omega} f^{2} \bar{u} d x+\int_{\Omega} f^{4} \bar{v} d x .
\end{aligned}
$$

Substituting $\overline{i \lambda u}$ given in (18) into $I_{1}$ and $\overline{i \lambda v}$ given in (20) into $I_{2}$, we have

$$
\begin{aligned}
& \int_{\Omega}|\nabla u|^{2} d x+\int_{\Omega}|\nabla v|^{2} d x+\alpha \int_{\Omega}(v \bar{u}+u \bar{v}) d x \\
& =\int_{\Omega}|\varphi|^{2} d x+\int_{\Omega}|\varphi|^{2} d x \\
& \quad+\int_{\Omega} \varphi \overline{f^{1}} d x+\int_{\Omega} \psi \overline{f^{3}} d x \\
& \quad+\int_{\Omega} f^{2} \bar{u} d x+\int_{\Omega} f^{4} \bar{v} d x .
\end{aligned}
$$

Using (32) our conclusion follows.

Lemma 4. The strong solution of the system (1)-(4) given by Theorem 1 satisfies

$$
\begin{aligned}
\left(1-\frac{c}{\lambda}\right) \int_{\Omega}|\psi|^{2} d x \leq & \frac{c}{|\lambda|}\left(\int_{\Omega}|\nabla v|^{2} d x+\int_{\Omega}|\nabla u|^{2} d x\right) \\
& +K|\lambda|^{2}\|U\|_{\mathscr{H}}\|F\|_{\mathscr{H}},
\end{aligned}
$$

where $K$ is a positive constant and $|\lambda|>1$ large enough.

Proof. Multiplying equality (21) by $\bar{\psi}$ and integrating on $\Omega$, we get

$$
\begin{aligned}
i \lambda \int_{\Omega}|\psi|^{2} d x= & \int_{\Omega} \Delta v \bar{\psi} d x-\alpha \int_{\Omega} u \bar{\psi} d x \\
& +\int_{\Omega} f^{4} \bar{\psi} d x .
\end{aligned}
$$

Using equality (20) and Poincaré and Young inequalities, we have

$$
\begin{aligned}
\int_{\Omega}|\psi|^{2} d x \leq & \frac{c}{|\lambda|}\left(\int_{\Omega}|\nabla v|^{2} d x+\int_{\Omega}|\nabla u|^{2} d x\right) \\
& +\frac{c}{|\lambda|} \int_{\Omega}|\psi|^{2} d x+K\|U\|_{\mathscr{H}}\|F\|_{\mathscr{H}}
\end{aligned}
$$

where $c$ is a positive constant and $|\lambda|>1$ is large enough. The proof is now complete.

Now, we are in the position to prove the main result of this paper. 
Theorem 5. The semigroup associated with the system (1)-(4) is polynomially stable and

$$
\left\|S(t) U_{0}\right\|_{\mathscr{H}} \leq \frac{K}{\sqrt{t}}\left\|U_{0}\right\|_{D(\mathscr{A})} .
$$

Moreover, this result is optimal.

Proof. From Lemmas 3 and 4 and for $|\lambda|>1$, we have

$$
\|U\|_{\mathscr{H}} \leq K|\lambda|^{2}\|F\|_{\mathscr{H}}
$$

which is equivalent to

$$
\left\|(\lambda I-\mathscr{A})^{-1}\right\| \leq K|\lambda|^{2}
$$

Then using Theorem 2.4 in [6], we obtain

$$
\left\|S(t) \mathscr{A}^{-1}\right\|=\mathcal{O}\left(t^{-1 / 2}\right) \Longrightarrow\left\|S(t) \mathscr{A}^{-1} F\right\|_{\mathscr{H}} \leq \frac{K}{\sqrt{t}}\|F\|_{\mathscr{H}} .
$$

Since $0 \in \rho(\mathscr{A})$, it follows that $\mathscr{A}$ is onto over $\mathscr{H}$; then taking $\mathscr{A} U_{0}=F$, we get

$$
\left\|S(t) U_{0}\right\|_{\mathscr{H}} \leq \frac{K}{\sqrt{t}}\left\|U_{0}\right\|_{D(\mathscr{A})} .
$$

Therefore the solution decays polynomially.

To prove that the rate of decay is optimal, we will argue by contradiction. Suppose that the rate $t^{-1 / 2}$ can be improved; for example, the rate is $t^{-1 /(2-\epsilon)}$ for some $0<\epsilon<2$. From Theorem 5.3 in [10], the operator

$$
|\lambda|^{-2+(\epsilon / 2)}\left\|(\lambda I-\mathscr{A})^{-1}\right\|_{\mathscr{L}(\mathscr{H})}
$$

should be limited, but this does not happen. For this, let us suppose that there exists a sequence $\left(\lambda_{\mu}\right) \subset \mathbb{R}$ with $\lim _{\mu \rightarrow \infty}\left|\lambda_{\mu}\right|=\infty$ and $\left(U_{\mu}\right) \subset D(\mathscr{A})$ for $\left(F_{\mu}\right) \subset \mathscr{H}$ such that

$$
\left(i \lambda_{\mu} I-\mathscr{A}\right) U_{\mu}=F_{\mu}
$$

is bounded in $\mathscr{H}$ and

$$
\lim _{\mu \rightarrow \infty}\left|\lambda_{\mu}\right|^{-2+(\epsilon / 2)}\left\|U_{\mu}\right\|_{\mathscr{H}}=\infty
$$

Then, we can consider, for each $\mu \in \mathbb{N}, F_{\mu}=\left(0, w_{\mu}, 0, w_{\mu}\right)^{T}$ and $U_{\mu}=\left(u_{\mu}, \varphi_{\mu}, v_{\mu}, \psi_{\mu}\right)^{T}$, where, due to the boundary conditions, $U_{\mu}$ are in the form $u_{\mu}=a w_{\mu}, v_{\mu}=b w_{\mu}, \varphi_{\mu}=c w_{\mu}$, and $\psi_{\mu}=d w_{\mu}$.

Then following the same steps as in the proof of Theorem 2 we can conclude that

$$
\left|\lambda_{\mu}\right|^{-2+(\epsilon / 2)}\left\|U_{\mu}\right\|_{\mathscr{H}} \geq O\left(\mu^{\epsilon / 2}\right) \longrightarrow \infty, \quad \text { as } \mu \longrightarrow \infty .
$$

Therefore the rate cannot be improved. The proof is now complete.

\section{Numerical Approaches}

In this section, we give a number of numerical experiments to certify the analytical results (see Figure 15). We use two numerical approaches to this aim: the spectral method to compute the numerical eigenvalues and the explicit time integration method to compute the numerical solutions and to derive the associated numerical energy. In particular, the numerical energy preserves the conservation/dissipation of energy. Moreover, by means of numerical simulations we can note a lack of numerical exponential decay considering only damping (see Figure 17).

Our numerical simulations are realized in the onedimensional case. Then we consider the following onedimensional system:

$$
\begin{array}{rr}
u_{t t}-u_{x x}+\alpha v+u_{t}=0, & \text { in }(0, L) \times(0, T), \\
v_{t t}-v_{x x}+\alpha u=0, & \text { in }(0, L) \times(0, T), \\
u(0, t)=u(L, t)=0, & v(0, t)=v(L, t)=0, \\
& 0<t<T, \\
u(\cdot, 0)=u_{0}(\cdot), & u_{t}(\cdot, 0)=u_{1}(\cdot), \\
v(\cdot, 0)=v_{0}(\cdot), & v_{t}(\cdot, 0)=v_{1}(\cdot) . \\
& \forall x \in(0, L) .
\end{array}
$$

4.1. Explicit Time Integration Method. For our purpose we define a computational mesh defining $\Delta x=L /(J+1)$ and $\Delta t=T /(N+1)$ for $J, N \in \mathbb{N}$ and the nets given by

$$
\begin{aligned}
& x_{0}=0<x_{1}=\Delta x<\cdots<x_{J}=J \Delta x<x_{J+1}=L, \\
& t_{0}=0<t_{1}=\Delta t<\cdots<t_{N}=N \Delta t<t_{N+1}=T,
\end{aligned}
$$

where $x_{j}=j \Delta x$ and $t_{n}=n \Delta t$ for $j=0,1,2, \ldots, J+1$ and $n=0,1,2, \ldots, N+1$.

We consider the following finite-difference discretization of (48)-(49):

$\frac{u_{j}^{n+1}-2 u_{j}^{n}+u_{j}^{n-1}}{\Delta t^{2}}-\frac{u_{j+1}^{n}-2 u_{j}^{n}+u_{j-1}^{n}}{\Delta x^{2}}+\frac{u_{j}^{n+1}-u_{j}^{n-1}}{2 \Delta t}+\alpha v_{j}^{n}=0$,

$$
\frac{v_{j}^{n+1}-2 v_{j}^{n}+v_{j}^{n-1}}{\Delta t^{2}}-\frac{v_{j+1}^{n}-2 v_{j}^{n}+v_{j-1}^{n}}{\Delta x^{2}}+\alpha u_{j}^{n}=0,
$$

for all $j=1,2, \ldots, J, n=1,2, \ldots, N$. Such scheme is in fact explicit and its implementation is straightforward. The boundary conditions are

$$
u_{0}^{n}=u_{J+1}^{n}=0, \quad v_{0}^{n}=v_{J+1}^{n}=0, \quad \forall n=1,2, \ldots, N,
$$


and the initial conditions are given by

$$
\begin{gathered}
u_{j}^{0}=u\left(x_{j}, 0\right), \quad u_{j}^{1}=u_{j}^{0}+\Delta t u_{t}\left(x_{j}, 0\right), \quad \forall j=1,2, \ldots, J, \\
v_{j}^{0}=v\left(x_{j}, 0\right), \quad v_{j}^{1}=v_{j}^{0}+\Delta t v_{t}\left(x_{j}, 0\right), \quad \forall j=1,2, \ldots, J .
\end{gathered}
$$

The discretizations above are consistent with truncation error of second order in $\Delta x$ and $\Delta t$. Therefore, it is immediate by Lax Lemma [11] that (53)-(54) converges. For convenience, we use the stability criterion (taking into account the conservative case) due to Courant-Friedrichs-Lewy; namely, $\Delta t \leq$ $\Delta x$.

4.2. Energy Conserving Scheme. In this section we will build the numerical energy to system (53)-(57). Following the techniques performed, for example, in $[12,13]$, this energy is given by

$$
\begin{aligned}
E^{n}:=\frac{\Delta x}{2} \sum_{j=0}^{J}[ & \left(\frac{u_{j}^{n+1}-u_{j}^{n}}{\Delta t}\right)^{2}+\left(\frac{u_{j+1}^{n+1}-u_{j}^{n+1}}{\Delta x}\right)\left(\frac{u_{j+1}^{n}-u_{j}^{n}}{\Delta x}\right) \\
& +\left(\frac{v_{j}^{n+1}-v_{j}^{n}}{\Delta t}\right)^{2}+\left(\frac{v_{j+1}^{n+1}-v_{j}^{n+1}}{\Delta x}\right)\left(\frac{v_{j+1}^{n}-v_{j}^{n}}{\Delta x}\right) \\
& \left.+\alpha\left(v_{j}^{n} u_{j}^{n+1}+u_{j}^{n} v_{j}^{n+1}\right)\right] .
\end{aligned}
$$

We note that $E^{n}$ is the discrete version of the continuous energy (5) in one-dimensional setting. Moreover, one can show that $E^{n}$ decreases. Instead of computing the time derivative of the energy we can use the summation by parts. The discrete energy $E^{n}$ is an important numerical instrument to certify our analytical results concerning the polynomial stabilization of system (53)-(57).

We have the following result.

Theorem 6 (discrete energy). Let $\left(u_{j}^{n}, v_{j}^{n}\right)$ be a solution of the finite difference scheme (53)-(57). Then for all $\Delta t$ and $\Delta x$, the discrete rate of change of energy of the numerical scheme (53)(57) at the $t_{n}$ instant of time is given by

$$
\frac{E^{n}-E^{n-1}}{\Delta t}=-\sum_{j=1}^{J}\left(\frac{u_{j}^{n+1}-u_{j}^{n-1}}{2 \Delta t}\right)^{2},
$$

for all $n=1, \ldots, N, N+1$.

Proof. We shortened the proof. It is identical to the proof of Proposition 1 in [12]. Then, we can write

$$
E^{n}+\Delta t \sum_{j=1}^{J}\left(\frac{u_{j}^{n+1}-u_{j}^{n-1}}{2 \Delta t}\right)^{2}=E^{n-1},
$$

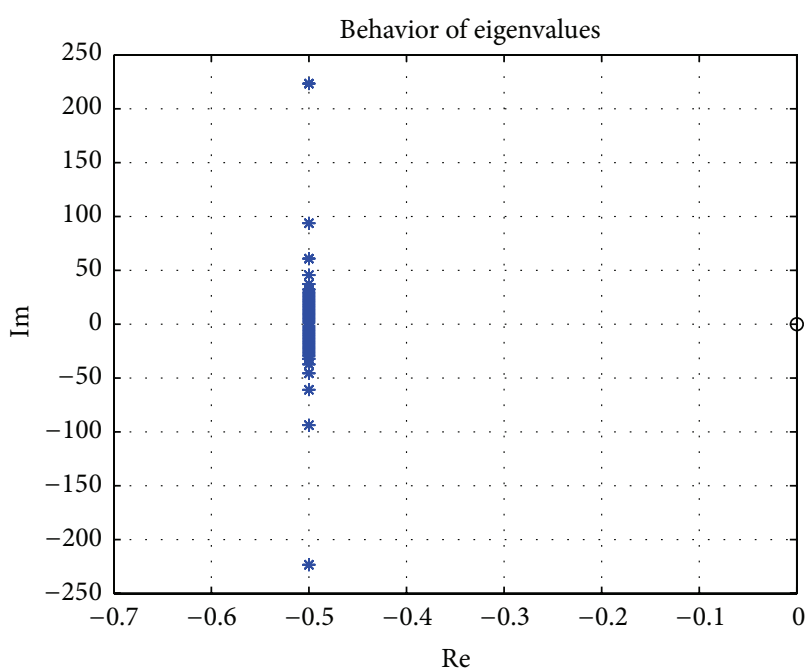

FIGURE 1: $\beta_{1}=1, \beta_{2}=1$.

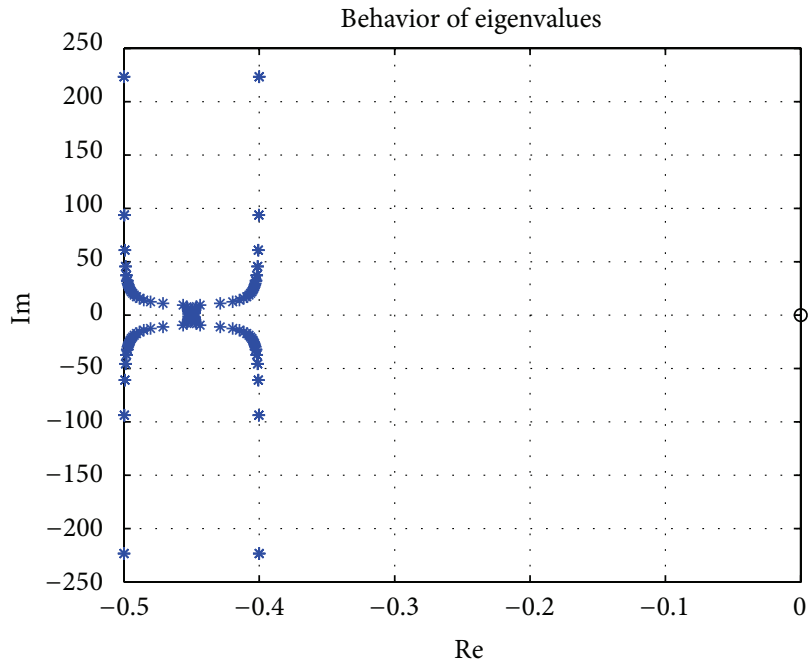

FIGURE 2: $\beta_{1}=1, \beta_{2}=0.8$.

from where we have

$$
E^{n} \leq E^{0}, \quad \forall n=1, \ldots, N, N+1 .
$$

Considering the conservative case we get

$$
E^{n}=E^{0}, \quad \forall n=1,2, \ldots, N .
$$

4.3. Discussion on Numerical Results. In this section, we consider two numerical approaches to certify the analytical results established in this work: the pseudospectral method to compute numerically the eigenvalues and also using the numerical energy $E^{n}$ in (58).

For spectral analysis we apply the spectral method according to Trefethen's book [14]. Using this numerical technique, we can see the numerical behavior of eigenvalues of (48)-(49). To see how this system is affected by a lack of 


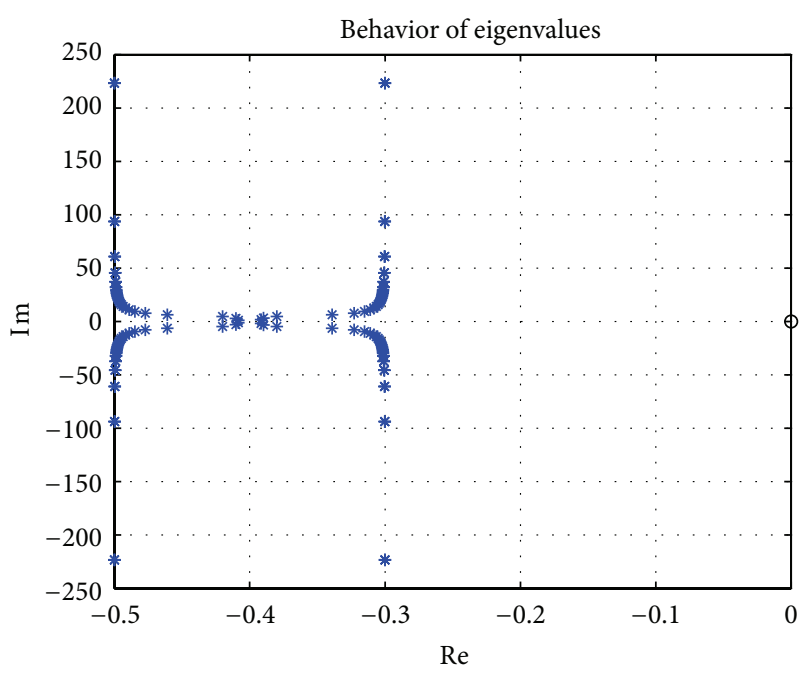

Figure 3: $\beta_{1}=1, \beta_{2}=0.6$.

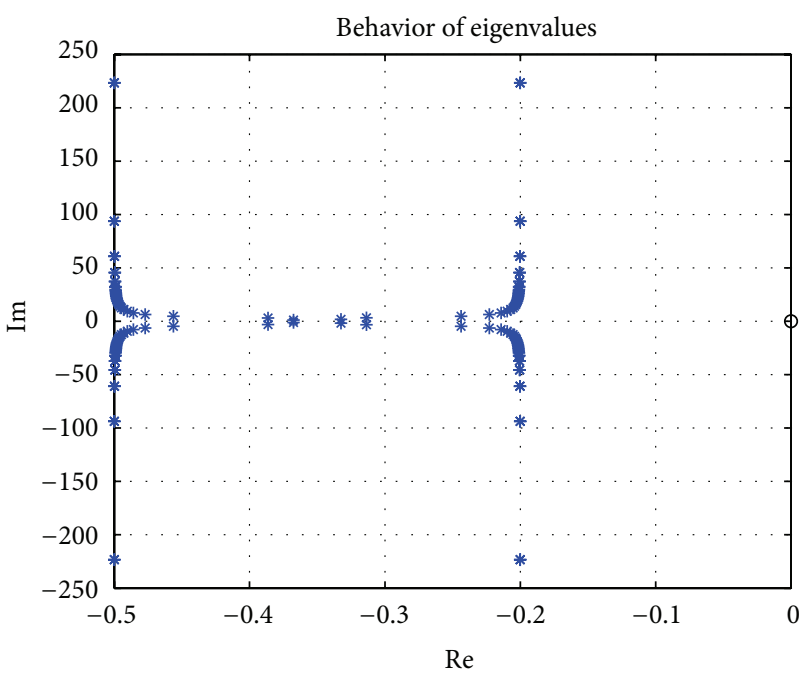

Figure $4: \beta_{1}=1, \beta_{2}=0.4$.

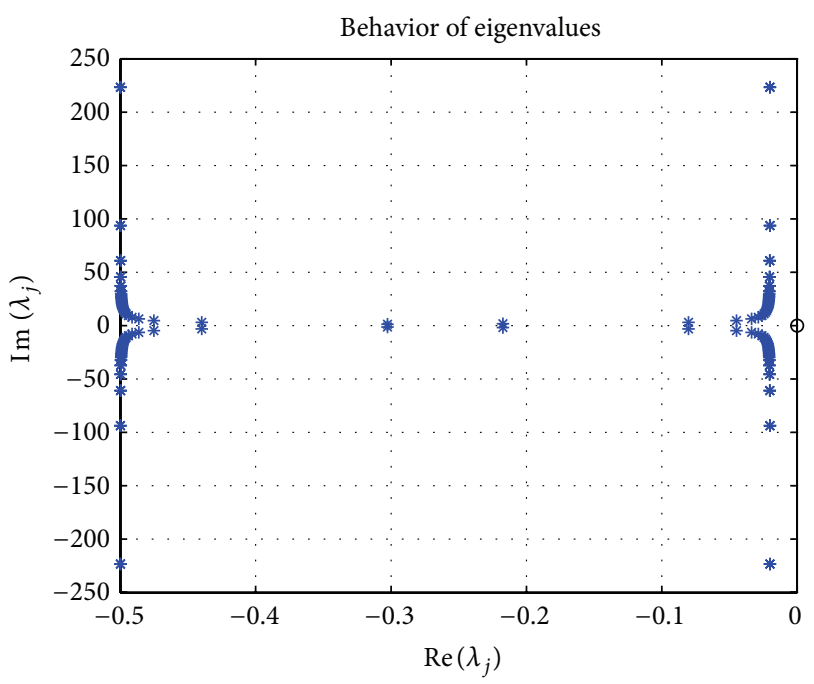

FIGURE 5: $\beta_{1}=1, \beta_{2}=0.04$.

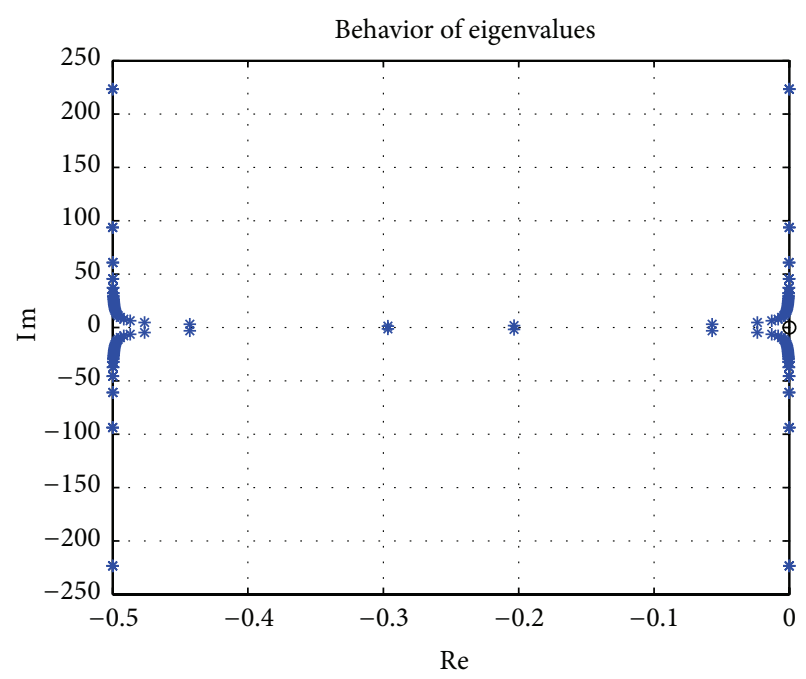

Figure 6: $\beta_{1}=1, \beta_{2}=0$.

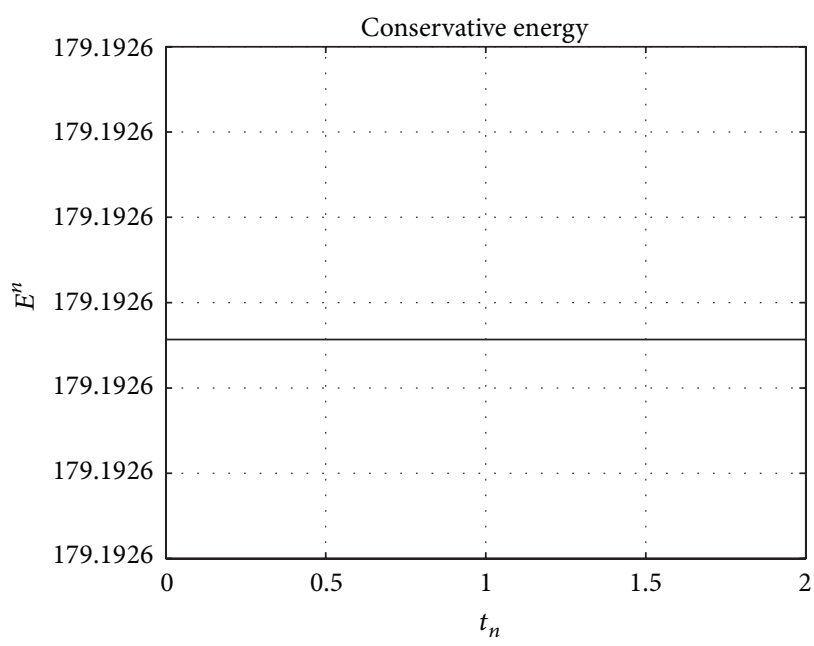

_ No damping

Figure 7: $\Delta t / \Delta x=0.9600$

exponential decay by means of several numerical simulations, we can consider two damping $\beta_{1} u_{t}$ and $\beta_{2} v_{t}$ where $\beta_{1}, \beta_{2} \geq 0$. Then, making $\beta_{2}$ go to zero, we can see a numerical sequence of eigenvalues converging to zero.

We consider $\beta_{1}=1$ and we take $\beta_{2}$ going to zero. Figures $1,2,3$, and 4 show that when $\beta_{i} \neq 0, i=1,2$, the real part of complex roots approaches negative asymptotes $-\beta_{i} / 2, i=$ 1,2 .

On the other hand, in Figures 5 and 6, we can see a sequence of eigenvalues converging to 0 as $\beta_{2} \rightarrow 0$.

Next, we show results of numerical simulations of scheme (53)-(57) and its energy $E^{n}$. Here we use the stability criterion $\Delta t \leq \Delta x$.

4.4. Undamped Case. Here, we consider $\alpha=0.25, L=1$, $T=2 \mathrm{~s}, u\left(x_{j}, 0\right)=v\left(x_{j}, 0\right)=2 \sin \left(3 \pi x_{j} / L\right), u_{t}\left(x_{j}, 0\right)=0$, 


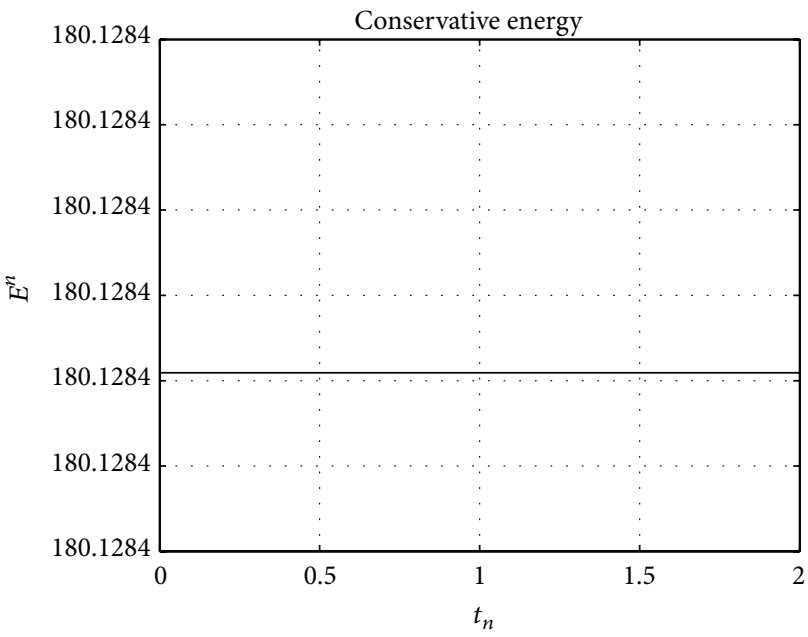

_ No damping

Figure 8: $\Delta t / \Delta x=0.8960$.

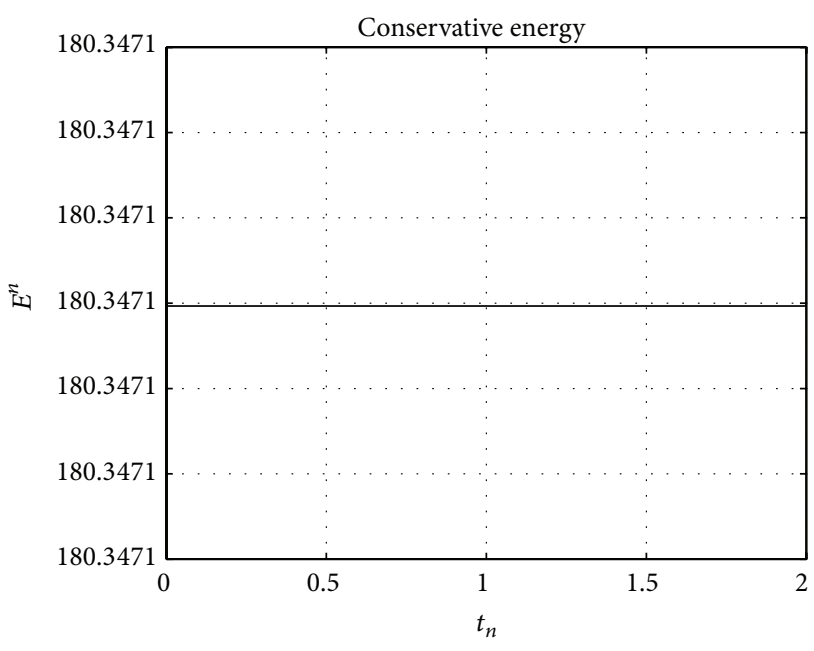

_ No damping

FIGURE 9: $\Delta t / \Delta x=0.7680$.

TABLE 1

\begin{tabular}{ccccc}
\hline & $\mu=0.9600$ & $\mu=0.8960$ & $\mu=0.7680$ & $\mu=0.7168$ \\
\hline$\varepsilon$ & 1.2103 & 0.2745 & 0.558 & 0.128 \\
\hline
\end{tabular}

and $v_{t}\left(x_{j}, 0\right)=3 \sin \left(3 \pi x_{j} / L\right)$. At continuous level we obtain that $E(0) \approx 180,4029$.

Figures 7, 8, 9, and 10 show the conservative behavior of numerical energy $E^{n}$ to undamped system. We can see the conservative behavior of the energy $E^{n}$ in agreement with Proposition 4.1. Moreover, it is clear that the accuracy of the approximations is achieved by means of refinements of type $\mu:=\Delta t / \Delta x \leq 1$.

A simple analysis, by using absolute error $\epsilon:=\left|E(0)-E^{0}\right|$ for each simulation, shows us the precision of the numerical energy. Table 1 shows the evolution of this error for each simulation.

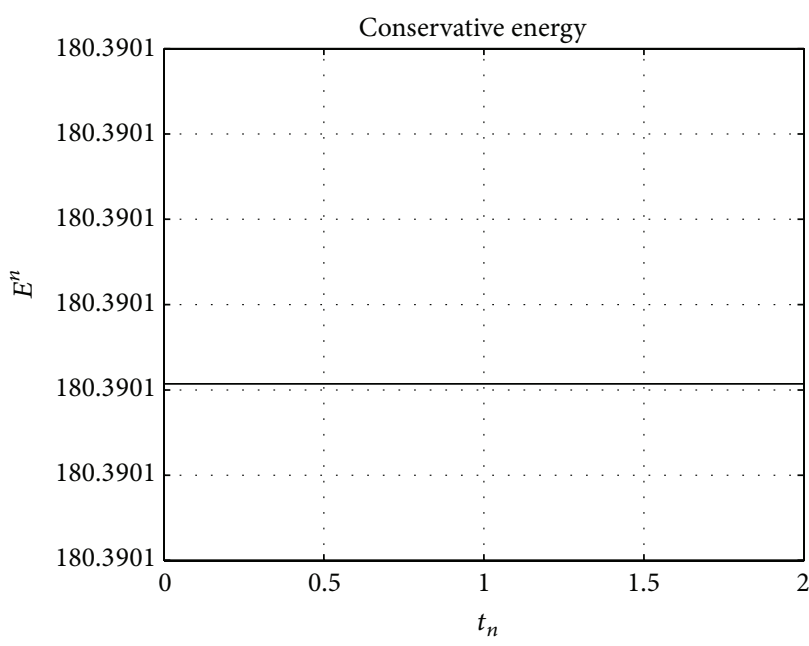

_ No damping

Figure 10: $\Delta t / \Delta x=0.7168$.

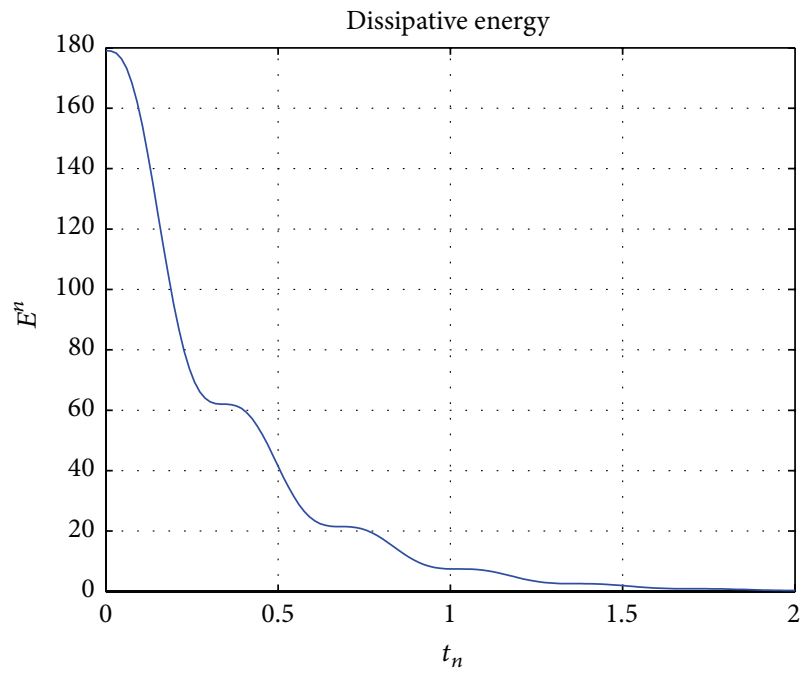

— Damping in $u$ and $v$

FIGURE 11: $\Delta t / \Delta x=0.9600$.

4.5. Full and Partial Damping. It is clear that considering two damping to the system (53)-(54) its numerical energy $E^{n}$ decays exponentially. See Figures 11 and 13. Therefore, in this case the energy $E^{n}$ is controllable by an exponential of type $e^{-\omega t_{n}}$ for some $\omega>0$. On the other hand, taking into account damping only one dissipative mechanism we observe a slow decay from value $\approx 180$ to value $\approx 90$ (see Figures 12 and 14 ). We can note a lack of 50 by cent in terms of exponential decay.

Note that the numerical solutions of the function $v$ do not go to the state of rest. See Figures 16 and 18.

\section{Conclusions}

In this work we considered a coupled system of two weakly dissipative wave equations. That is to say, the damping mechanism acts partially on the system and it is well known that this 


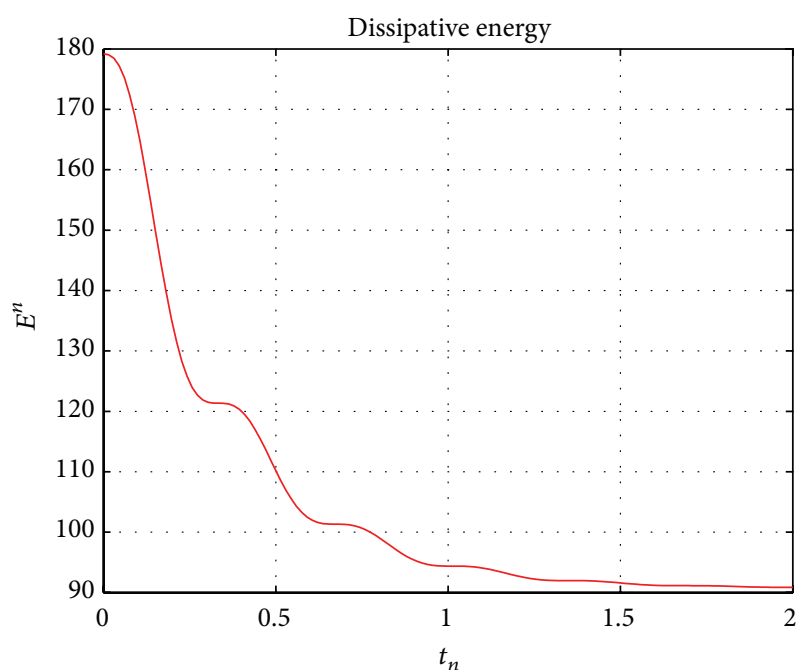

_ Damping only $u$

FIGURE 12: $\Delta t / \Delta x=0.960$.

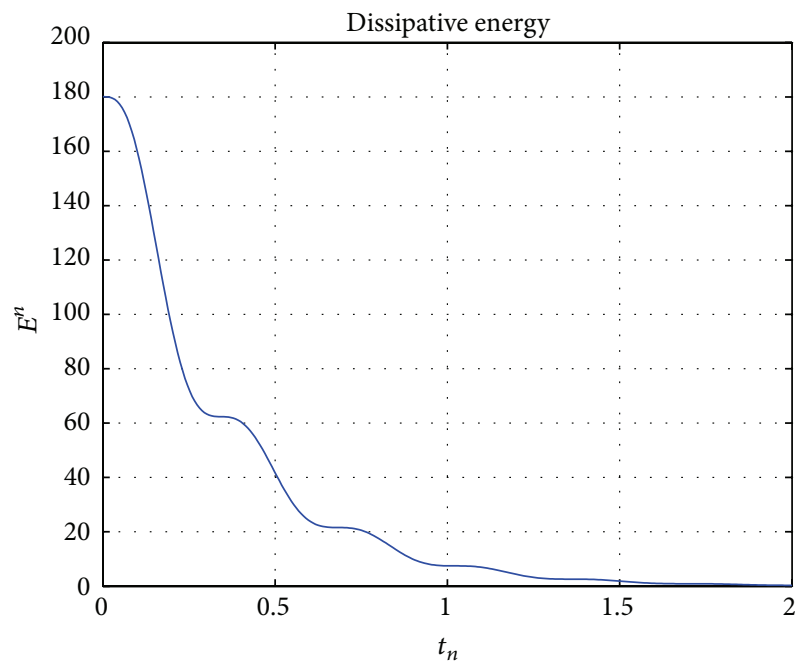

_ Damping in $u$ and $v$

Figure 13: $\Delta t / \Delta x=0.8960$.

system is not exponentially stable. Our main contribution is twofold: the optimality of the polynomial decay in accordance with a recent theoretical contribution due to Borichev and Tomilov [6] and its numerical certification by using finite differences method. In particular, we derive a numerical energy and we show the lack of exponential decay by means of several numerical experiments.

The analytical and numerical techniques performed in this work can be applied to systems where there exists a lack of exponential decay. See, for example, the work of Santos et al. for examples of systems with the property of lack of exponential decay and polynomial decay.

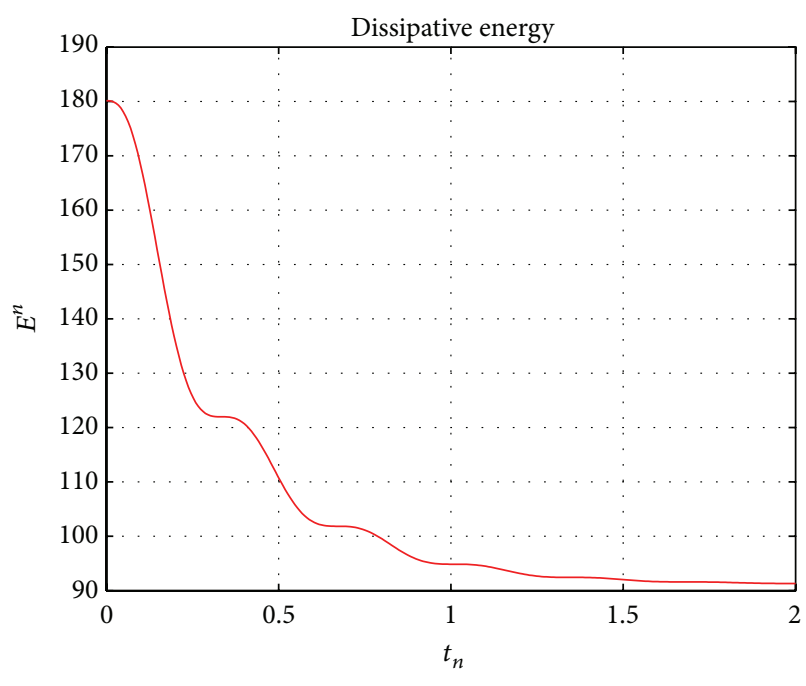

_ Damping only $u$

FIGURE 14: $\Delta t / \Delta x=0.8960$.

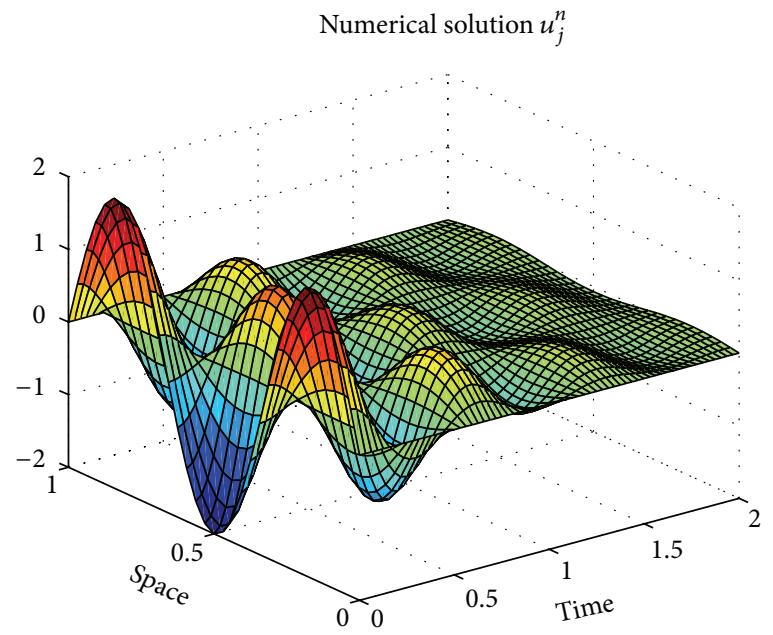

Figure 15: Partial damping.

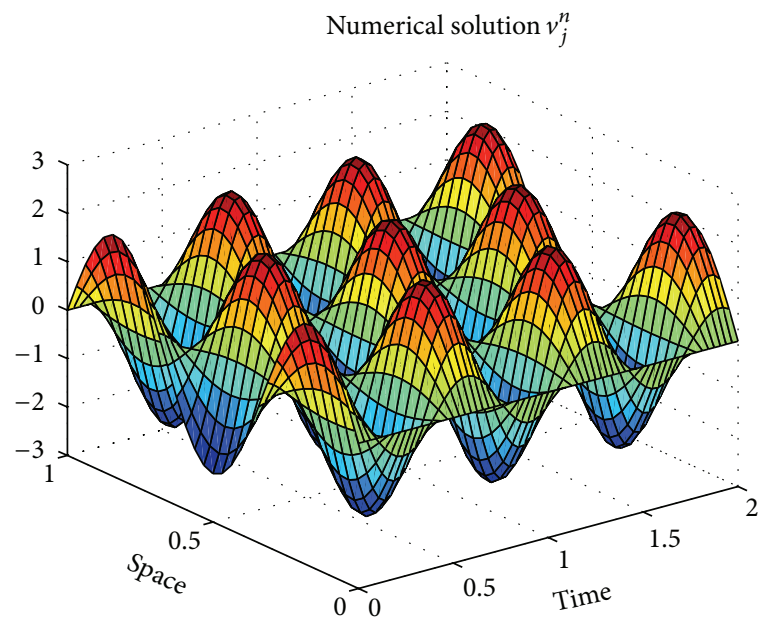

Figure 16: Partial damping. 


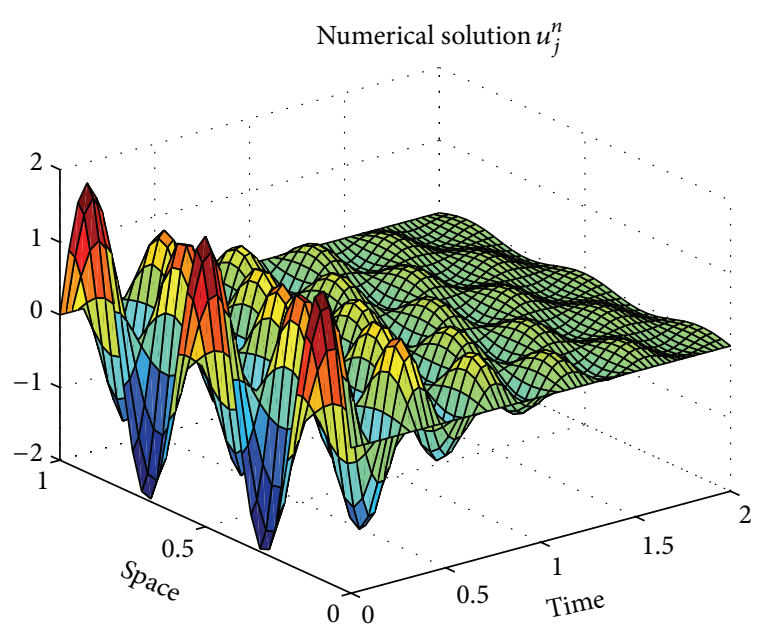

FIgURE 17: Partial damping.

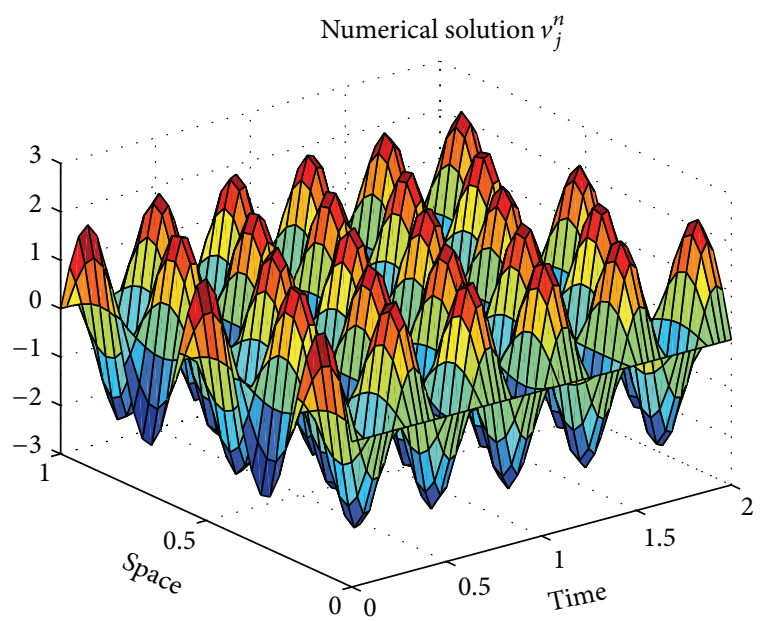

FIgURE 18: Partial damping.

\section{Conflict of Interests}

The authors declare that there is no conflict of interests regarding the publication of this paper.

\section{Acknowledgments}

The authors would like to acknowledge the support of the following corporations: UFPA, PROPESP, and FADESP through PAPQ. The authors thank the anonymous referees for their valuable comments as well as reference suggestions that helped to improve the presentation of this paper.

\section{References}

[1] M. Najafi, G. R. Sarhangi, and H. Wang, "The study of stability of coupled wave equations under various end conditions," in Proceedings of 31st Conferences on Decision and Control, pp. 374379, Tucson, Arizona, 1992.
[2] V. Komornik and B. Rao, "Boundary stabilization of compactly coupled wave equations," Asymptotic Analysis, vol. 14, no. 4, pp. 339-359, 1997.

[3] F. Alabau Boussoira, "Stabilisation frontière indirecte de systèmes faiblement couplés," Comptes Rendus de l'Académie des Sciences. Série I, vol. 328, no. 11, pp. 1015-1020, 1999.

[4] F. Alabau Boussoira, P. Cannarsa, and V. Komornik, "Indirect internal stabilization of weakly coupled evolution equations," Journal of Evolution Equations, vol. 2, no. 2, pp. 127-150, 2002.

[5] M. L. Santos, M. P. C. Rocha, and S. C. Gomes, "Polynomial stability of a coupled system of wave equations weakly dissipative," Applicable Analysis, vol. 86, no. 10, pp. 1293-1302, 2007.

[6] A. Borichev and Y. Tomilov, "Optimal polynomial decay of functions and operator semigroups," Mathematische Annalen, vol. 347, no. 2, pp. 455-478, 2010.

[7] M. Najafi, "Study of exponential stability of coupled wave systems via distributed stabilizer," International Journal of Mathematics and Mathematical Sciences, vol. 28, no. 8, pp. 479-491, 2001.

[8] A. Pazy, Semigroups of Linear Operators and Applications to Partial Differential Equations, Springer, New York, NY, USA, 1983.

[9] J. Prüss, "On the spectrum of $C_{0}$-semigroups," Transactions of the American Mathematical Society, vol. 284, no. 2, pp. 847-857, 1984.

[10] L. H. Fatori and J. E. M. Rivera, "Rates of decay to weak thermoelastic Bresse system," IMA Journal of Applied Mathematics, vol. 75, no. 6, pp. 881-904, 2010.

[11] G. D. Smith, Numerical Solution of Partial Differential Equations: Finite Difference Methods, Oxford Applied Mathematics and Computing Science Series, 1984.

[12] M. Negreanu and E. Zuazua, "Uniform boundary controllability of a discrete 1-D wave equation," Systems \& Control Letters, vol. 48, no. 3-4, pp. 261-279, 2003.

[13] W. Strauss and L. Vazquez, "Numerical solution of a nonlinear Klein-Gordon equation," Journal of Computational Physics, vol. 28, no. 2, pp. 271-278, 1978.

[14] L. N. Trefethen, Spectral Methods in MATLAB, SIAM, Philadelphia, Pa, USA, 2000. 


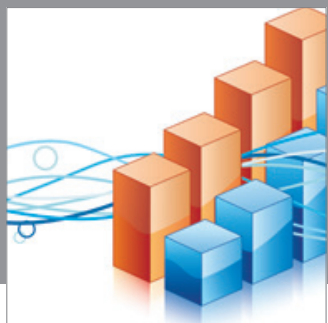

Advances in

Operations Research

mansans

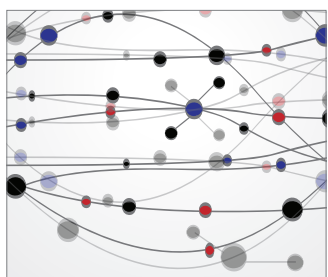

The Scientific World Journal
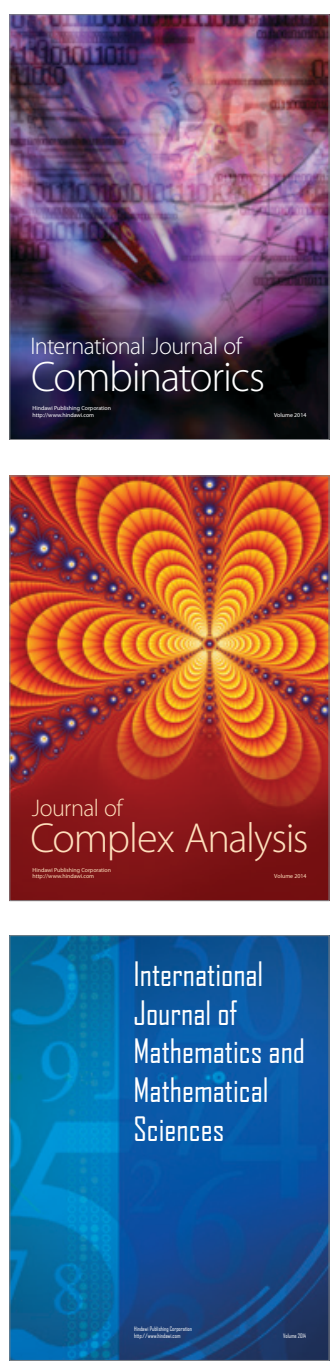
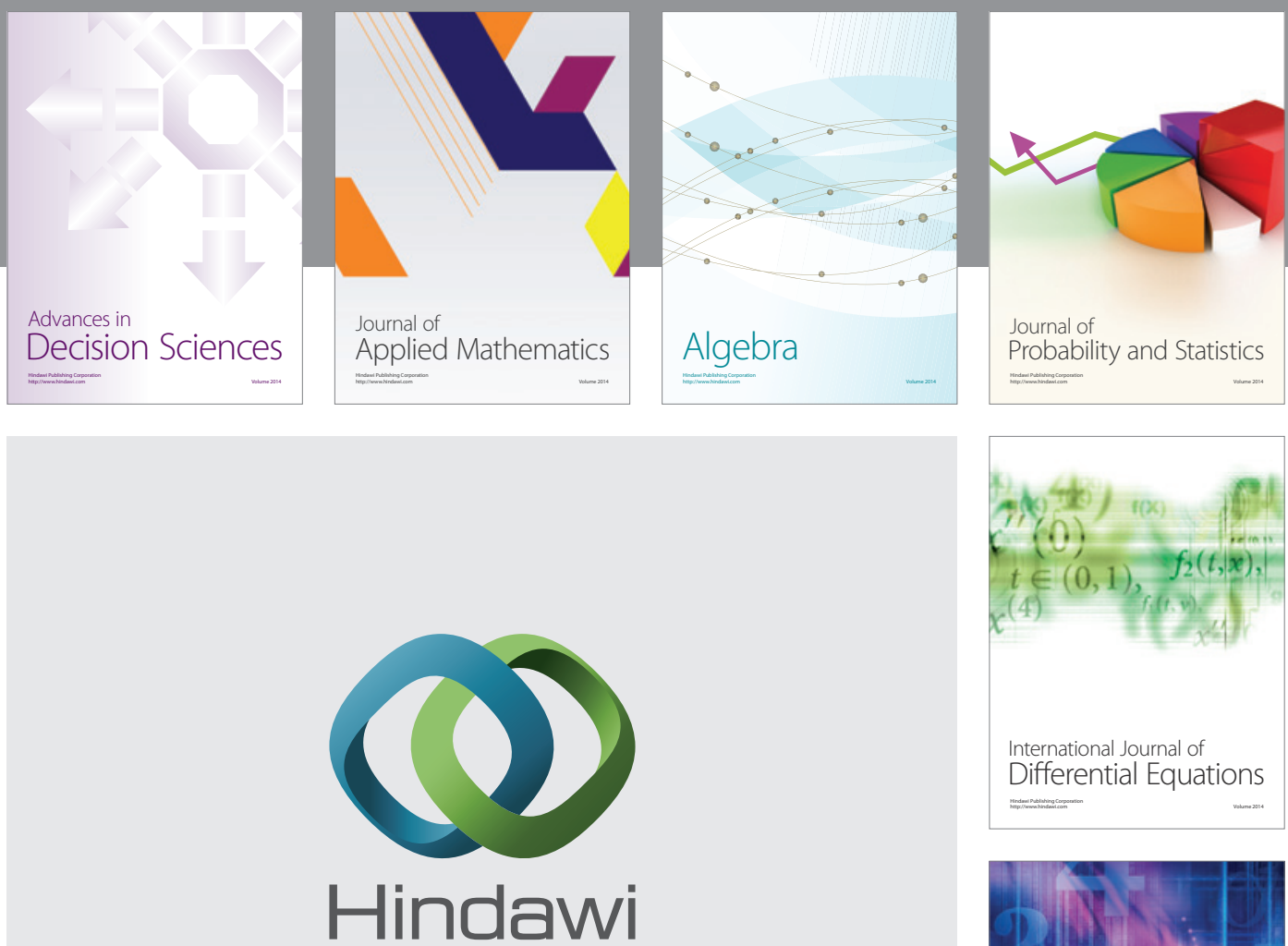

Submit your manuscripts at http://www.hindawi.com
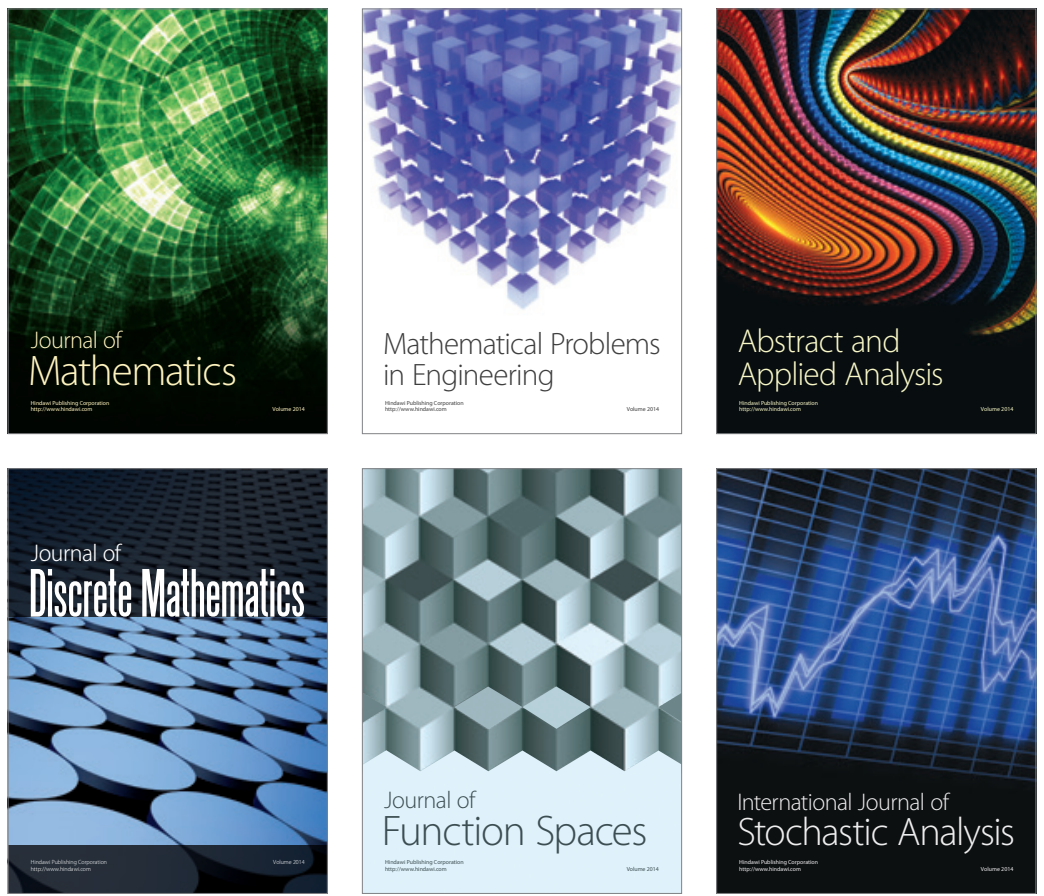

Journal of

Function Spaces

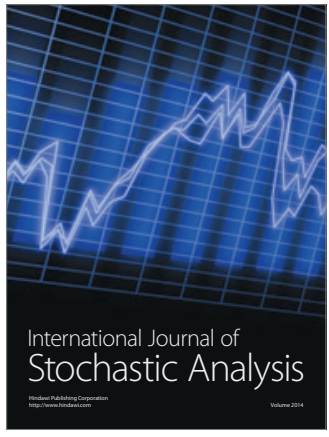

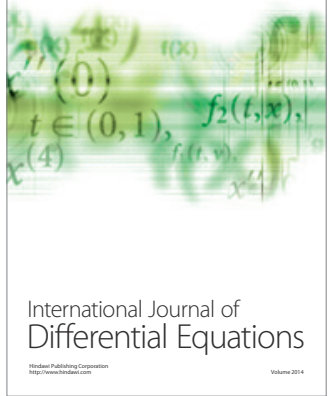
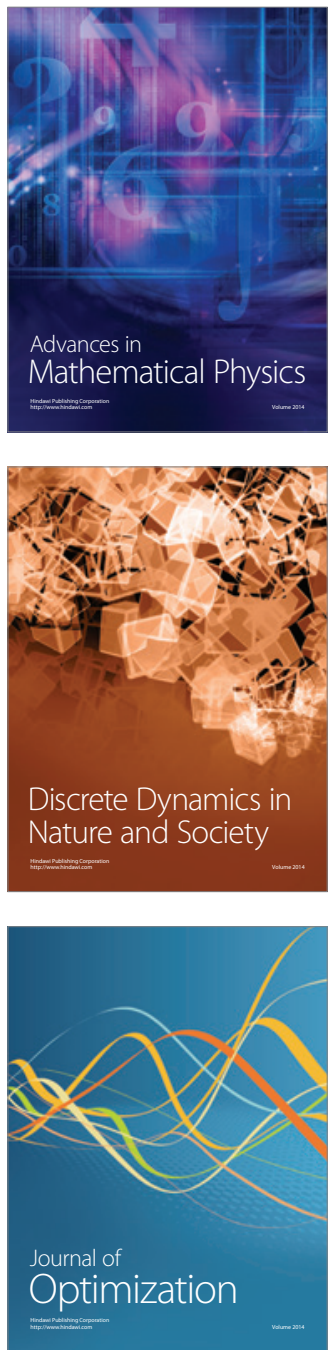